\title{
CARBONO EM AGREGADOS DO SOLO SOB VEGETAÇÃO NATIVA, PASTAGEM E SISTEMAS AGRÍCOLAS NO BIOMA CERRADO $^{(1)}$
}

\author{
Ciniro Costa Junior ${ }^{(2)}$, Marisa de Cássia Píccolo ${ }^{(3)}$, Marcos Siqueira Neto ${ }^{(4)}$, Plínio \\ Barbosa de Camargo $^{(5)}$, Carlos Clemente Cerri ${ }^{(6)} \&$ Martial Bernoux $^{(7)}$
}

\section{RESUMO}

A matéria orgânica do solo (MOS) é responsável pela fertilidade, ciclagem de nutrientes e estabilidade da estrutura, possuindo estreita relação com a agregação do solo. No entanto, ainda não é clara a relação entre as classes de agregados na dinâmica de acúmulo ou perda de MOS em função do uso e manejo do solo. Este trabalho teve como objetivo avaliar os teores de carbono (C) em agregados do solo sob quatro usos e manejos: Cerrado nativo (CE), sistema plantio direto (SPD), sistema plantio convencional (SPC) e pastagem (PA). As áreas de estudo estão localizadas no município de Rio Verde (GO), em solo classificado como Latossolo Vermelho distrófico de textura argilosa. Em amostras de solo coletadas em fevereiro de 2007 nas camadas de 0-5, 5-10 e 10-20 cm de profundidade, foram determinados: a quantidade de macroagregados (maiores que 2,0 mm), mesoagregados (maiores que 0,25 e menores que $2,00 \mathrm{~mm}$ ) e microagregados (maiores que 0,05 e menores que $0,25 \mathrm{~mm}$ ) estáveis em água, os índices de agregação diâmetro médio ponderado (DMP) e geométrico (DMG) e os teores de $\mathrm{C}$ nas classes de agregados estabelecidas. No solo avaliado observou-se predominância de macroagregados, com maior quantidade sob CE e PA, em comparação ao solo sob SPD e SPC, indicando efeito negativo do cultivo do solo na conservação de macroagregados. Contudo, os teores de $\mathbf{C}$ nos agregados do solo na camada de $0-20 \mathrm{~cm}$ foram maiores no CE $\mathrm{e}$ SPD em relação a PA e SPC, sugerindo que o não revolvimento e a manutenção dos resíduos culturais na superfície do solo favorecem o acúmulo de C. Com isso, foi verificado

(1) Parte da Dissertação de Mestrado do primeiro autor ao Programa de Pós-Graduação em Ciências - CENA/USP. Recebido para publicação em 19 de outubro de 2010 e aprovado em 6 de junho de 2012.

(2) Doutorando do Laboratório de Biogeoquímica Ambiental. Centro de Energia Nuclear na Agricultura, Universidade de São Paulo - CENA/USP. CP 96, CEP 13400-970, Piracicaba (SP). E-mail: cinirojr@cena.usp.br

(3) Pesquisadora do Laboratório de Ciclagem de Nutrientes do CENA/USP. E-mail: mpiccolo@cena.usp.br

(4) Pós-doutorando do Laboratório de Biogeoquímica Ambiental do CENA/USP. E-mail: msiqueir@gmail.com

(5) Pesquisador do Laboratório de Ecologia Isotópica do CENA/USP. E-mail: pcamargo@cena.usp.br

(6) Pesquisador do Laboratório de Biogeoquímica Ambiental do CENA/USP. E-mail: cerri@cena.usp.br

(7) Pesquisador do IRD, UMR Eco\&Sols (SupAgro, Cirad, Inra, IRD), 2 Place Viala - Bat. 12, 34060 Montpellier Cedex 2. France. E-mail: martial.bernoux@ird.fr 


\begin{abstract}
que os macro e mesoagregados podem ser mais sensíveis ao tipo de uso e manejo do solo, quando comparados aos microagregados. A agregação do solo sob PA é semelhante à do $\mathrm{CE}$, embora com menores teores de $\mathrm{C}$, sugerindo dinâmica de agregação diferenciada, o que merece a atenção de novas pesquisas.
\end{abstract}

Termos de indexação: matéria orgânica do solo, agregação do solo, uso e manejo do solo.

\title{
SUMMARY: CARBON IN SOIL AGGREGATES UNDER NATIVE VEGETATION, PASTURE AND AGRICULTURAL SYSTEMS IN THE BRAZILIAN SAVANNAH
}

\begin{abstract}
Soil organic matter (SOM) is responsible for soil fertility, nutrient cycling and structure stability and is closely related with soil aggregation. However, the relationship between aggregate size classes in the SOM dynamics is unclear, resulting in SOM accumulation or loss due to soil land use and management. This study aimed to evaluate the amount of carbon $(C)$ in soil aggregates under four uses and management: native Cerrado (CE-savannah vegetation), notillage (NT), conventional tillage (SPC) and pasture (PA). The study areas are located in Rio Verde (GO) where the soil was classified as kaolinitic clayey Oxisol. The soil layers 0-5, 5-10 and 10-20 cm were sampled $(n=6)$ in February 2007, and the amount of water-stable macroaggregates $(>2.0 \mathrm{~mm})$, mesoaggregates $(>0.25$ and $<2.00 \mathrm{~mm}$ ) and microaggregates (> 0.05 and $<0.25 \mathrm{~mm}$ ) were determined, as well as the mean weight diameter (MWD) and mean geometric diameter (MGD), and the C levels in the soil aggregate classes. Macroaggregates were predominant in the soil evaluated, with higher quantities in CE and PA compared NT and SPC soils, indicating a negative effect of soil cultivation on macroaggregate conservation. However, the soil aggregate C levels in the 0-20 cm layer were higher in CE and SPD than in PA and SPC soils, suggesting that the absence of tillage and maintenance of crop residues on the soil drives $C$ accumulation. This shows that macroaggregates and mesoaggregates were more sensitive to soil use and management than microaggregates. The similarities of aggregation in $P A$ and $C E$ soils, although with lower C levels in PA suggested different aggregation dynamics, requiring further research.
\end{abstract}

Index terms: soil organic matter, soil aggregation, soil use and management.

\section{INTRODUÇÃO}

O tipo de cultivo do solo pode causar mudanças em sua agregação; uma relação causal com a matéria orgânica do solo (MOS) tem sido evidenciada em diversas pesquisas (An et al., 2010; Anders et al., 2010; Fernandez et al., 2010; Huang et al., 2010).

O conteúdo de MOS é aceito como agente de formação e estabilização dos agregados do solo (Six et al., 2004; Noellemeyer et al., 2008; Mulumba \& Lal, 2008), considerando ainda que o aumento de seu teor deveria ser prioridade não somente para o sequestro de C, mas também para aumento da qualidade, fertilidade, ciclagem de nutrientes e estabilidade da estrutura do solo (Li et al., 2007; Barreto et al., 2009).

Stevenson (1994) sugeriu que substâncias orgânicas, principalmente as húmicas, formam um filme sobre as partículas de solo, cimentando-as em agregados estáveis. Os microagregados são mantidos unidos por materiais orgânicos persistentes e substâncias poliméricas que, associados a materiais temporários, como raízes de plantas e hifas de fungos, formam e estabilizam os macroagregados. Estudos reportam que a presença de macroagregados é, de modo geral, associada positivamente com os conteúdos de MOS (Green et al., 2005; De Gryze et al., 2008; Anders et al., 2010), os quais ainda protegem o solo contra a degradação e erosão pela água da chuva, sobretudo em zonas tropicais e subtropicais (Bayer et al., 2006; Noellemeyer et al., 2008).

Muitos desses estudos mostram que a adoção do sistema plantio direto (SPD) conduz a maiores quantidades de MOS se comparado ao sistema plantio convencional (SPC) (Six \& Jastrow, 2002; Ogle et al., 2003; Salton et al., 2008; Fabrizzi et al., 2009; Anders et al., 2010), concomitantemente com a formação de macroagregados (Pinheiro et al., 2004; Six et al., 2006). O aumento do estoque de C sob SPD é frequentemente atribuído ao maior conteúdo de material orgânico particulado adicionado aos primeiros centímetros do perfil do solo e ao menor distúrbio deste, comparado ao SPC, os quais são importantes fatores que intervêm na estabilização dos macroagregados (Six et al., 2000; Fabrizzi et al., 2003; Madari et al., 2005). 
No cultivo de gramínea em pastagens também existe relação entre aumento da agregação e teores de $\mathrm{C}$ no solo. Por possuírem sistema radicular abundante e de rápido crescimento, as gramíneas são capazes de agrupar fisicamente as partículas de solo e, em conjunto com a liberação de exsudatos, estimulam a cimentação dessas partículas e, consequentemente, 0 acúmulo de C (Bronick \& Lal, 2005; Salton et al., 2008).

Entretanto, segundo Fernández et al. (2010), ainda não é claro qual classe de agregados é responsável pelo acúmulo de $\mathrm{C}$ e quais tipos de solos, camadas ou fatores nos manejos são decisivos nesse processo. Comparados a solos com argila do tipo 2:1, solos com argila do tipo 1:1 (solos de Cerrado) apresentam quantidade maior de macroagregados $(>2,00 \mathrm{~mm})$, com menor correlação com a MOS (Six et al., 2004). Isso se deve à formação de ligações eletrostáticas entre partículas primárias e secundárias do solo, principalmente óxidos, que atuam na formação e estabilização desses agregados (Muggler et al., 1999). Nesse caso, agentes biológicos de agregação do solo possuem papel secundário nesse processo. Apesar do manejo realizado em situações climáticas semelhantes, os solos 2:1 apresentam maior perda de C, denotando ser mais vulneráveis às mudanças de uso e manejo do solo (Fabrizzi et al., 2009).

Considerando as classes de agregados, em recente estudo, Fernández et al. (2010) mostraram que macroagregados $(>2,00 \mathrm{~mm})$ e microagregados $(0,25>0,05 \mathrm{~mm})$ são suscetíveis ao distúrbio físico do solo, porém apenas nos agregados de tamanho intermediário é que se refletem as diferenças na dinâmica do $\mathrm{C}$ sob diferentes sistemas de manejo do solo.

Assim, mais pesquisas são necessárias para elucidar os mecanismos e a sensibilidade devido às mudanças ambientais de manejo na agregação do solo associada à MOS (Fabrizzi et al., 2009; Fernández et al., 2010).

No Brasil, cerca de metade dos 2 milhões de $\mathrm{km}^{2}$ originais do Cerrado foram transformados em pastagens, culturas anuais e outros tipos de uso, sendo os solos desse bioma altamente intemperizados. Objetivou-se, com este estudo, verificar a influência de usos do solo (vegetação nativa, pastagem e agricultura), além de dois manejos agrícolas (sistema convencional e plantio direto), na agregação e nos teores de $\mathrm{C}$ de um Latossolo Vermelho distrófico caulinítico de Cerrado no Centro-Oeste do Brasil.

\section{MATERIAL E MÉTODOS}

As áreas de estudo estão localizadas no município de Rio Verde, Estado de Goiás. O clima da região, segundo Köppen, é do tipo Aw tropical, com chuvas concentradas no verão e período seco bem definido durante a estação de inverno. A média anual da precipitação pluvial é de $1.500 \mathrm{~mm}$, e a média anual da temperatura, de $23{ }^{\circ} \mathrm{C}$ (Figura 1). $\mathrm{O}$ solo foi classificado como Latossolo Vermelho distrófico caulinítico de textura argilosa (Embrapa, 2006), com vegetação original predominantemente arbórea, com fitofisionomia de Cerradão e mata seca subcaducifólia (Einten, 1972).

Este trabalho compreende parte de um estudo maior que teve como objetivo geral avaliar as alterações nos estoques de $\mathrm{C}$ do solo com o tempo de implantação do SPD. Para isso, entre abril de 2001 e outubro de 2002 foi realizada a seleção de áreas com potencial interesse para a realização do estudo (Siqueira Neto et al., 2010). Inicialmente, o levantamento de seleção das áreas foi constituído de entrevistas com proprietários e gerentes em fazendas da região, a fim de reunir informações sobre o uso da terra e tipificar os manejos empregados.

A análise dessas entrevistas indicou que o manejo com maior predominância foi o Sistema Plantio Direto cultivado com soja (Glycine max [L.] Merr.) como cultura comercial principal, sucedida por milho (Zea mays L.), sorgo (Sorghum bicolor [L.] Moench) ou milheto (Pennisetum glaucum [L.] R. Br.) em safrinha.

Nas fazendas selecionadas foram realizadas amostragens de solo georreferenciadas, com o intuito de conhecer previamente o $\mathrm{pH}$, a textura, o material de origem e a classificação do solo nas áreas. Foi também utilizada a base de dados do projeto RadamBrasil (Brasil, 1981), com informações sobre geologia, geomorfologia, pedologia, vegetação e uso potencial da terra, com escala de 1:1.000.000. Esse conjunto de informações foi combinado com técnicas de sensoriamento remoto e, dessa forma, buscou-se maior homogeneidade no conjunto de informações levantadas para a seleção das áreas.

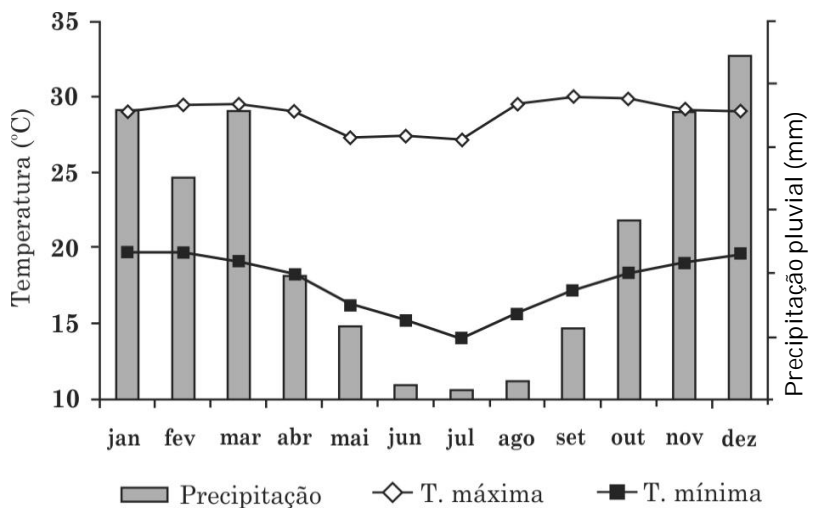

Figura 1. Temperatura média mensal (máxima e mínima) $\left({ }^{\circ} \mathrm{C}\right)$ e precipitação pluvial média $(\mathrm{mm})$ no bioma Cerrado, em Rio Verde (GO). Os dados são referentes ao período de 1972 a 2004 . Fonte: Cooperativa Mista dos Produtores Rurais do Sudoeste Goiano Ltda (COMIGO). 
Para seleção final das áreas, foram considerados o histórico, o manejo empregado, a homogeneidade nas características do solo (material de origem, textura e classe), bem como o critério de menor distância entre os pontos amostrais. Do total de 251 áreas localizadas e analisadas, foram selecionadas 12 delas, com teor de argila entre 50 e $70 \%$, das quais quatro foram avaliadas no presente estudo, sendo: uma área sob Vegetação Nativa com fitofisionomia de Cerradão - CE ( $17^{\circ}$ 39' 07" de latitude sul e $51^{\circ} 02{ }^{\prime} 49^{\prime \prime}$ de longitude oeste), com estrato arbóreo semidecidual e altura entre 15 a $25 \mathrm{~m}$; uma área sob pastagem - PA ( $17^{\circ} 38^{\prime} 58^{\prime \prime}$ de latitude sul e $51^{\circ} 03$ ' $57^{\prime \prime}$ de longitude oeste) que, após o desmatamento, foi cultivada com arroz (Oryza sativa L.) por nove anos, com posterior introdução da pastagem com braquiária (Brachiaria decumbens Stapf) por 20 anos - aplicação de $3,0 \mathrm{Mg}^{-1}$ de calcário dolomítico antes da implantação da pastagem e $80 \mathrm{~kg} \mathrm{ha}^{-1}$ de NPK (04-20-18) a cada três anos; uma área sob sistema plantio convencional - SPC $\left(17^{\circ} 41^{\prime} 36^{\prime \prime}\right.$ de latitude sul e $51^{\circ} 10^{\prime} 56^{\prime \prime}$ de longitude oeste) que, após o desmatamento, foi cultivada com arroz (Oryza sativa L.) por dois anos, seguido por soja (Glycine $\max \mathrm{L}$. Merr.) alternada com milho, anualmente, por 29 anos - foram aplicados $2,0 \mathrm{Mg} \mathrm{ha}^{-1}$ de calcário dolomítico dois anos antes da realização do estudo; e uma área sob sistema plantio direto - SPD $\left(17^{\circ} 20^{\prime} 12^{\prime \prime}\right.$ de latitude sul e $51^{\circ} 15^{\prime} 03^{\prime \prime}$ de longitude oeste) que, após o desmatamento, foi convertida em pastagem por cinco anos, seguida pelo cultivo de arroz em sistema convencional durante dois anos, depois milho e soja, alternadamente, por oito anos e posteriormente convertido para plantio direto, por 15 anos, de soja sucedida por milho ou sorgo (Sorghum bicolor L. Moench). Para esta última, foram aplicados $3,0 \mathrm{Mg} \mathrm{ha}^{-1}$ de calcário dolomítico em superfície, seis anos antes da realização deste estudo; contudo, para manutenção dos teores de $\mathrm{Ca}$ e $\mathrm{Mg}$, foram aplicados $400 \mathrm{~kg} \mathrm{ha}^{-1}$ de silicato de cálcio e magnésio no ano anterior à realização do estudo.

Nas áreas sob SPD e SPC, a soja recebeu aplicação de $450 \mathrm{~kg} \mathrm{ha}^{-1}$ do formulado 02-20-18 na semeadura e uma cobertura de $100 \mathrm{~kg} \mathrm{ha}^{-1}$ de KCl realizada aos 22 dias após a semeadura, obtendo-se produtividade média de grãos de $3.600 \mathrm{~kg} \mathrm{ha}^{-1}$. A safrinha de milho foi realizada apenas na área cultivada em SPD, na qual foram aplicados $550 \mathrm{~kg} \mathrm{ha}^{-1} \mathrm{de} \mathrm{NPK}$ (08-20-20) na semeadura e cobertura de $200 \mathrm{~kg} \mathrm{ha}^{-1}$ de ureia, com produtividade média de grãos de $7.500 \mathrm{~kg} \mathrm{ha}^{-1}$.

Para melhor caracterização, em cada área foi feita a descrição do perfil pedológico até $1,5 \mathrm{~m}$ de profundidade. As características verificadas foram a cor (Munsell, 1954), os teores de argila, silte e areia (Embrapa, 1997), a densidade do solo (Blake \& Hartge, 1986) e a composição mineralógica determinada em um difrator de raios X da marca Phillips PW-1830.

Portanto, mesmo que as áreas estejam espacialmente dispersas, pode-se assumir que o meio atuou uniformemente em todas as unidades experimentais, uma vez que os atributos do solo são facilmente identificáveis como homogêneos para receber os tratamentos.

No quadro 1 estão apresentados os resultados das principais características dos horizontes diagnósticos $\mathrm{A}$ e $\mathrm{Bw}_{1}$ em perfis de Latossolo Vermelho distrófico caulinítico nas áreas que compõem este estudo e, no quadro 2, as características físico-químicas das áreas na camada de $0-20 \mathrm{~cm}$ de solo.

Em fevereiro de 2007, as áreas de estudo (CE, PA, SPC e SPD) foram amostradas, empregando-se um método estatístico de análise de dados inteiramente casualizados (DIC) com seis repetições $(n=6)$, por meio da escavação de trincheiras ( $50 \mathrm{~cm}$ de profundidade, $1,5 \mathrm{~m}$ de comprimento e 1,0 $\mathrm{m}$ de largura) e retirada de monólitos de solo das camadas de 0-5, 5-10 e 10$20 \mathrm{~cm}$, com a ajuda de espátulas.

Para obtenção dos agregados e determinação da estabilidade, utilizou-se o método descrito por Kemper \& Chepil (1965), com alterações propostas por Silva \& Mielniczuk (1997), que consiste basicamente no fracionamento do monólito de solo observando-se os pontos de fraqueza, para que o volume total da amostra transpassasse a malha de $9,51 \mathrm{~mm}$, sendo excluídos dela fragmentos de plantas, outros resíduos, pedras e cascalhos retidos na peneira. A separação dos agregados em classes de tamanho foi feita pela desagregação e peneiramento em meio úmido. Foram obtidas três classes de agregados: macroagregados (diâmetro $>2,00 \mathrm{~mm}$ ), mesoagregados (diâmetro entre 2,00 e $0,25 \mathrm{~mm}$ ) e microagregados (diâmetro entre 0,25 e $0,05 \mathrm{~mm}$ ). Os valores obtidos nos peneiramentos foram usados para cálculo do diâmetro médio geométrico (GMP) por meio da equação: DMP = $\sum_{\mathrm{i}=1}^{\mathrm{n}}(x i . w i)$, em que $w i=$ proporção $(\%)$ de cada classe em relação ao total e $x i=$ diâmetro médio das classes, expresso em mm; e diâmetro médio geométrico (DMG) por meio da equação: $\mathrm{DMG}=10^{\mathrm{W}}$, sendo $\mathrm{w}=[\Sigma(\mathrm{z} \log \mathrm{y}$ $/ \Sigma \mathrm{n}]$, segundo equações propostas por Kemper \& Chepil (1965).

Os índices DMP e DMG podem sintetizar as informações relacionadas à distribuição dos agregados do solo, permitindo comparar diversos sistemas de manejo quanto à organização da estrutura do solo (Salton et al., 2008). Para as determinações dos teores de $\mathrm{C}$ total, uma alíquota de cada classe de agregados do solo obtida foi totalmente moída e passada em peneiras de malha de $0,150 \mathrm{~mm}$, com posterior pesagem de $0,150 \mathrm{~g}$ dessa alíquota para análise por combustão a seco no equipamento LECO ${ }^{\circ} \mathrm{CN}-2000$. Todas as análises foram feitas no Laboratório de Biogeoquímica Ambiental, no Centro de Energia Nuclear na Agricultura/USP (Piracicaba - SP).

Os resultados foram submetidos às pressuposições da análise de variância (homogeneidade de variância e normalidade dos dados) e as médias comparadas pelo teste de Tukey $(p<0,05)$; para as correlações de Pearson foi aplicado o teste $t(p<0,01$ e 0,05$)$, utilizando o programa SAS (2003). 
Quadro 1. Características dos horizontes diagnósticos A e Bw1 em perfis de Latossolo Vermelho distrófico caulinítico sob diferentes usos e manejos do solo, no município de Rio Verde (GO)

\begin{tabular}{|c|c|c|c|c|}
\hline Característica & $\mathbf{C E}$ & PA & SPC & SPD \\
\hline & \multicolumn{4}{|c|}{ Horizonte A } \\
\hline Cor (úmida) ${ }^{a}$ & $2,5 \mathrm{YR} 3 / 3$ & $2,5 \mathrm{YR} 3 / 3$ & $5 \mathrm{YR} 3,5 / 3$ & $2,5 \mathrm{YR} 3 / 3$ \\
\hline Profundidade $(\mathrm{cm})$ & $0-6$ & $0-17$ & $0-13$ & $0-11$ \\
\hline Densidade $\left(\mathrm{g} \mathrm{cm}^{-3}\right)$ & 0,87 & 1,23 & 1,03 & 0,96 \\
\hline Areia $\left(\mathrm{g} \mathrm{kg}^{-1}\right)^{\mathrm{b}}$ & 414 & 274 & 397 & 248 \\
\hline Silte $\left(\mathrm{g} \mathrm{kg}^{-1}\right)^{\mathrm{c}}$ & 45 & 45 & 42 & 87 \\
\hline \multirow[t]{2}{*}{ Argila $\left(\mathrm{g} \mathrm{kg}^{-1}\right)^{\mathrm{c}}$} & 541 & 681 & 561 & 665 \\
\hline & \multicolumn{4}{|c|}{ Horizonte Bw1 } \\
\hline Cor (úmida) & $2,5 \mathrm{YR} 3 / 6$ & $2,5 \mathrm{YR} 3 / 6$ & $2,5 \mathrm{YR} 4 / 7$ & $2,5 \mathrm{YR} 3 / 6$ \\
\hline Profundidade $(\mathrm{cm})$ & $36-79$ & $36-76$ & $24-75$ & $36-72$ \\
\hline Densidade $\left(\mathrm{g} \mathrm{cm}^{-3}\right)$ & 1,23 & 1,21 & 1,11 & 1,05 \\
\hline Areia $\left(\mathrm{g} \mathrm{kg}^{-1}\right)$ & 313 & 303 & 350 & 262 \\
\hline Silte $\left(\mathrm{g} \mathrm{kg}^{-1}\right)$ & 69 & 56 & 34 & 60 \\
\hline Argila $\left(\mathrm{g} \mathrm{kg}^{-1}\right)$ & 628 & 641 & 616 & 678 \\
\hline \multicolumn{5}{|l|}{ Mineralogia $^{d}$} \\
\hline Quartzo e $^{\text {e }}$ & +++ & +++ & +++ & +++ \\
\hline Caolinita & ++++ & ++++ & ++++ & ++++ \\
\hline Gibsita & +++ & +++ & +++ & +++ \\
\hline Hematita & ++ & ++ & ++ & +++ \\
\hline Anatásio & + & + & + & + \\
\hline
\end{tabular}

CE: Cerrado nativo; SPC: sistema plantio convencional com 29 anos; SPD: sistema plantio direto com 15 anos; PA: pastagem com 20 anos (previamente cultivada sob SPC por 9 anos).

${ }^{\mathrm{a}}$ Cor úmida (Carta de Munsell, 1954); ${ }^{\mathrm{b}}$ Teores de areia determinados por peneiramento úmido (Embrapa, 1979); ${ }^{\mathrm{c}}$ Teores de silte e argila determinados pelo método do densímetro após dispersão em hexametafosfato e digestão da matéria orgânica em $\mathrm{H}_{2} \mathrm{O}_{2}$; ${ }^{\mathrm{d}}$ Composição mineralógica determinada por difração de raios $\mathrm{X} ;{ }^{\mathrm{e}}+=$ intensidade do sinal e quantidade de picos apresentada no espectro das amostras.

Quadro 2. Características físico-químicas de um Latossolo Vermelho distrófico sob diferentes usos e manejos do solo, em Rio Verde (GO), na camada de 0-20 cm

\begin{tabular}{|c|c|c|c|c|c|c|c|c|c|c|}
\hline \multirow{2}{*}{$\begin{array}{l}\text { Usos e manejos } \\
\text { do solo }\end{array}$} & \multicolumn{3}{|c|}{ Granulometria } & \multicolumn{7}{|c|}{ Propriedades químicas } \\
\hline & Areia & Silte & Argila & pH $\mathbf{H}_{2} \mathrm{O}$ & $\mathbf{P}$ & $\mathbf{K}$ & $\mathbf{C a}$ & $\mathbf{M g}$ & CTC & $\mathbf{V}$ \\
\hline & & \multicolumn{2}{|l|}{$\mathrm{g} \mathrm{kg}^{-1}$} & \multicolumn{3}{|c|}{$\mathrm{mg} \mathrm{kg}^{-1}$} & \multicolumn{2}{|c|}{$\mathrm{mmol}_{\mathrm{c}} \mathrm{dm}^{3}$} & & $\%$ \\
\hline $\mathrm{CE}$ & 413 & 46 & 541 & 4.7 & 6.8 & 1,1 & 1,0 & 1,3 & 82,6 & 4,12 \\
\hline $\mathrm{PA}$ & 311 & 47 & 641 & 6.1 & 8.3 & 0,9 & 11,2 & 11,0 & 53,1 & 43,50 \\
\hline SPC & 330 & 56 & 614 & 5.5 & 11.3 & 2,2 & 10,0 & 2,7 & 49,4 & 30,16 \\
\hline SPD & 309 & 53 & 638 & 6.0 & 29.3 & 1,7 & 15,3 & 6,2 & 62,8 & 36,94 \\
\hline
\end{tabular}

CE: Cerrado nativo; SPC: sistema plantio convencional com 29 anos; SPD: sistema plantio direto com 15 anos; PA: pastagem com 20 anos (previamente cultivada sob SPC por 9 anos). 


\section{RESULTADOS E DISCUSSÃO}

\section{Agregação do solo}

A distribuição das classes de agregados do solo estáveis em água indicou predominância de macro e mesoagregados (em torno de $90 \%$ ) para as três camadas estudadas (Figura 2). Esses resultados ilustram a capacidade dos solos de regiões de clima tropical de apresentar elevada influência de interações eletrostáticas dos óxidos e minerais de argila 1:1 no processo de agregação (Six et al., 2004; Zotarelli et al., 2007; Fabrizzi et al., 2009). Da mesma forma, Salton et al. (2008) verificaram elevada quantidade dessas classes de agregados em Latossolos do bioma Cerrado ao compararem sistemas de manejo do solo, incluindo lavouras em plantio direto, lavouras (soja) em rotação com pastagens em sistema plantio direto e pastagens permanentes (Brachiaria sp), no Mato Grosso do Sul.

Partindo do pressuposto de que o estado de agregação máxima ocorre em solos sob vegetação nativa (Six et al., 2002), foi observado que os solos manejados com sucessão de cultivos apresentaram maior quantidade de meso e microagregados e, consequentemente, menores valores de DMP e DMG, possivelmente devido à ruptura dos macroagregados (Quadro 3 e Figura 2).

Apenas na camada superficial do solo $(0-5 \mathrm{~cm})$ a quantidade de macroagregados foi maior no SPD, quando comparada à área sob SPC e demais usos do solo avaliados (Figura 2). Nessa camada de solo, os valores de DMP e DMG em SPD foram cerca de $40 \%$ superiores aos observados na área sob plantio convencional (Quadro 3).

Esses resultados indicam que os agregados de maior tamanho seriam os mais alterados pela destruição mecânica atribuída ao uso de implementos mecânicos de preparo que empregam o revolvimento do solo e que o estado de maior agregação do solo sob SPD em relação ao SPC foi devido, além do não revolvimento, à manutenção dos resíduos vegetais (soja/milho) na superfície, principalmente na camada $0-5 \mathrm{~cm}$ de solo, na qual o contato com os resíduos culturais é maior em relação às camadas mais profundas (Pinheiro et al., 2004; Mulumba \& Lal, 2008; Barreto et al., 2009; Martins et al., 2009).

Madari et al. (2005) encontraram padrão similar ao observado neste estudo, com maior quantidade de macroagregados sob SPD em relação ao SPC apenas na camada de $0-5 \mathrm{~cm}$ de um Latossolo Vermelho eutroférrico na Mata Atlântica, em uma floresta semidecídua no Sul do Brasil. Semelhantemente, Pinheiro et al. (2004) encontraram maiores valores de macroagregados, DMP e DMG sob pastagem (Panicum maximum L.) (70, 4,2 e 1,1\%, respectivamente), seguido do SPD (50, 3,0 e 1,0\%, respectivamente), e menores no SPC (30, 2,0 e 1,0\%, respectivamente) na camada de 0-5 cm de um Latossolo Vermelho distrófico de Mata Atlântica, no Rio de Janeiro. Também Corrêa (2002), em Latossolo Vermelho-Amarelo no bioma Cerrado $(0-15 \mathrm{~cm})$, relatou valores significativamente maiores de percentagem de macroagregados e DMP sob SPD (36 e 2,1\%, respectivamente), em relação ao $\mathrm{SPC}(23$ e $1,6 \%$, respectivamente).

Por outro lado, a área sob PA apresentou macroagregados, mesoagregados, DMP e DMG semelhantes aos do solo sob vegetação nativa (CE) em todas as camadas avaliadas (Figura 2 e Quadro 4). Esse fato evidenciou a capacidade das pastagens de manter a agregação do solo, por meio do efeito do crescimento das raízes no processo de agregação, que estimula a atividade microbiana e, consequentemente, aumenta a quantidade de exsudatos que atuam como agentes de agregação do solo, além de forças mecânicas que estabilizam agregados com maior intensidade em comparação a culturas como soja e milho (Denef \& Six, 2005; Salton et al., 2008).

Quadro 3. Índices de agregação diâmetro médio ponderado (DMP) e diâmetro médio geométrico (DMG) de um Latossolo Vermelho distrófico sob diferentes usos e manejos do solo, em Rio Verde (GO)

\begin{tabular}{|c|c|c|c|c|c|c|}
\hline \multirow[t]{3}{*}{ Usos e manejos do solo } & \multicolumn{3}{|c|}{ DMP } & \multicolumn{3}{|c|}{ DMG } \\
\hline & \multicolumn{6}{|c|}{ Camada de solo $(\mathrm{cm})$} \\
\hline & $0-5$ & $5-10$ & $10-20$ & $0-5$ & $5-10$ & $10-20$ \\
\hline $\mathrm{CE}$ & $4,02 \mathrm{a}^{*}$ & 4,06 a & $4,02 \mathrm{a}$ & $2,69 \mathrm{a}$ & $2,75 \mathrm{a}$ & $2,72 \mathrm{a}$ \\
\hline $\mathrm{PA}$ & $3,87 \mathrm{a}$ & $3,94 \mathrm{a}$ & $3,94 \mathrm{a}$ & $2,49 \mathrm{a}$ & $2,49 \mathrm{a}$ & $2,41 \mathrm{a}$ \\
\hline SPC & $2,27 \mathrm{c}$ & $2,99 \mathrm{~b}$ & $3,17 \mathrm{~b}$ & $1,09 \mathrm{c}$ & $1,58 \mathrm{~b}$ & $1,68 \mathrm{~b}$ \\
\hline SPD & $3,46 \mathrm{~b}$ & $3,06 \mathrm{~b}$ & $2,96 \mathrm{~b}$ & $1,76 \mathrm{~b}$ & $1,56 \mathrm{~b}$ & $1,46 \mathrm{~b}$ \\
\hline
\end{tabular}

CE: Cerrado nativo; SPC: sistema plantio convencional com 29 anos; SPD: sistema plantio direto com 15 anos; PA: pastagem com 20 anos (previamente cultivada sob SPC por 9 anos). DMP e DMG: diâmetro médio ponderado e geométrico dos agregados do solo, respectivamente. Letras iguais nas colunas não diferem a $5 \%$. pelo teste de Tukey. *Valores representam a média $(\mathrm{n}=6)$. 


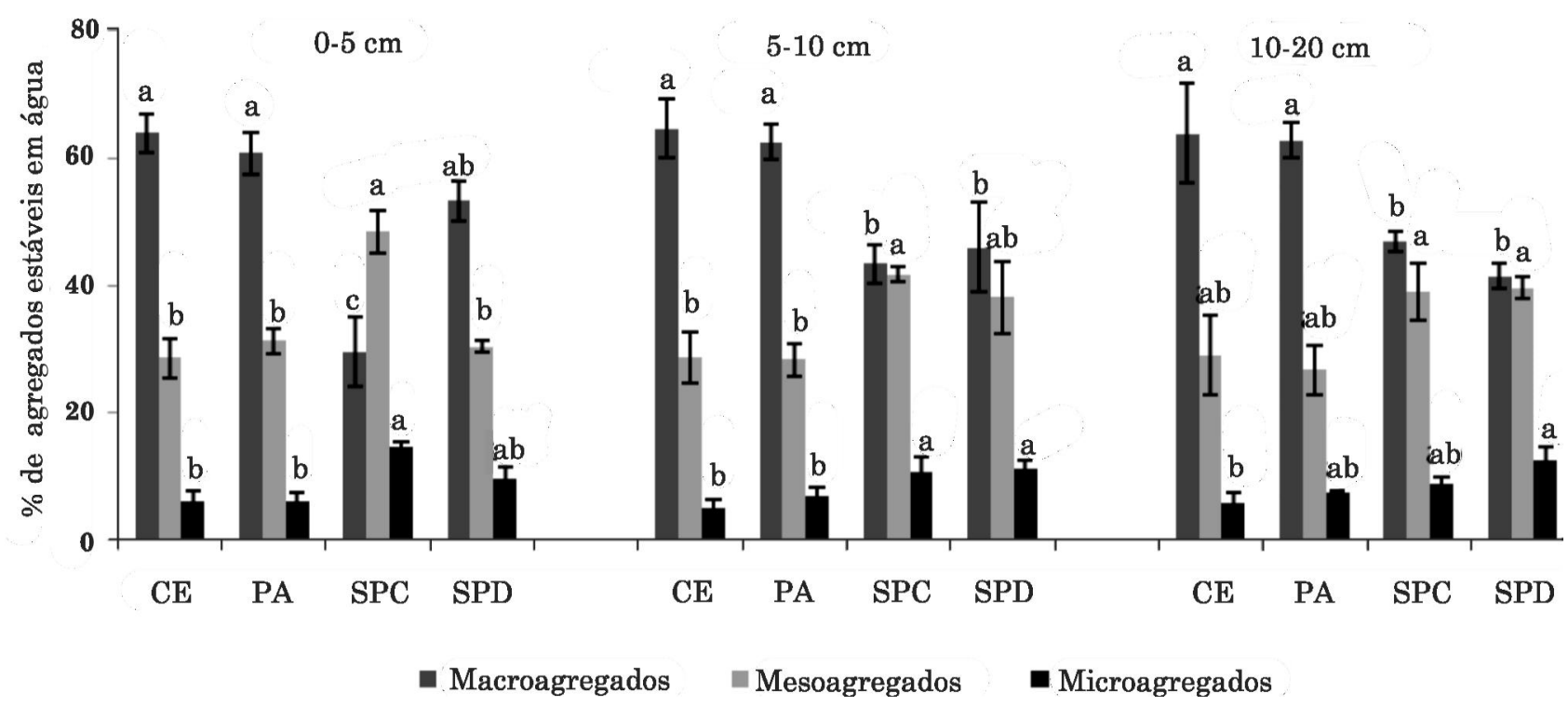

Figura 2. Distribuição relativa de agregados estáveis em água nas camadas de solo de 0-5, 5-10 e 10-20 cm (n=6) de um Latossolo Vermelho distróficos sob diferentes usos e manejos do solo, em Rio Verde (GO). CE: Cerrado nativo; SPC: sistema plantio convencional com 29 anos; SPD: sistema plantio direto com 15 anos; PA: pastagem com 20 anos (previamente cultivada sob SPC por 9 anos). Macro, meso e microagregados: agregados com diâmetro $>2,00 \mathrm{~mm}$, entre 2,00 e $0,25 \mathrm{~mm}$ e 0,25 e $0,05 \mathrm{~mm}$, respectivamente. Letras iguais para a mesma classe de agregados e camada de solo não diferem a $5 \%$ pelo teste de Tukey.

Salton et al. (2008) destacaram que a manutenção do DMP com valores semelhantes ou superiores aos verificados em Latossolo Vermelho distrófico no Bioma Cerrado em um Cerradão somente foi observada sob rotação lavoura-pastagem em ciclos de dois anos ou com pastagem permanente, devido à elevada influência do sistema radicular na agregação do solo. Isso se deve à ação mecânica decorrente do crescimento e funcionamento das raízes, dos microrganismos e da fauna do solo, que são estimulados com a presença de pastagem, proporcionando o agrupamento dos microagregados, o que resulta na formação de macroagregados (Haynes \& Beare, 1997).

\section{Teores de $\mathrm{C}$ nos agregados do solo}

Em todas as áreas avaliadas, na camada de 0-5 cm de solo os mesoagregados apresentaram os maiores teores de $\mathrm{C}$ em relação aos macro e microagregados. Nas demais camadas, os microagregados apresentaram os menores valores, exceto sob SPD, cujos valores foram semelhantes entre as classes (Quadro 4).

Considerando a camada de $0-20 \mathrm{~cm}$, foram verificadas sob $\mathrm{CE}$ as maiores médias, correspondentes aos macro e mesoagregados, com teores de $\mathrm{C}$ de $39,9 \mathrm{e}$ $40,9 \mathrm{~g} \mathrm{~kg}^{-1}$, respectivamente, exceto nos microagregados, com $25,1 \mathrm{~g} \mathrm{~kg}^{-1}$. Nos demais tratamentos, o SPD $\left(37,8,36,1\right.$ e $\left.35,1 \mathrm{~g} \mathrm{~kg}^{-1}\right)$ apresentou valores superiores aos de PA (30,3, 31,3 e $\left.23,4 \mathrm{~g} \mathrm{~kg}^{-1}\right)$ e SPC $\left(31,9,33,6\right.$ e $\left.25,5 \mathrm{~g} \mathrm{~kg}^{-1}\right)$ em todas as classes de agregados (Quadro 4 ).
Esses resultados indicaram que o SPD foi capaz de promover teores de $\mathrm{C}$ similares aos encontrados no solo sob vegetação original (CE) e até mesmo maiores nos microagregados, demonstrando que esse sistema incorporou mais $\mathrm{C}$ no solo em relação às práticas do SPC e da pastagem avaliadas na profundidade de 0 $20 \mathrm{~cm}$ (Quadro 4).

A expressiva tendência do solo sob SPD em atingir valores de $\mathrm{C}$ similares aos da área nativa $(\mathrm{CE})$ vem sendo frequentemente reportada na literatura (Six et al., 1998, 2000; Pinheiro et al., 2004; Madari et al., 2005; Barreto et al., 2009). Pinheiro et al. (2004), em um Latossolo Vermelho distrófico no Estado do Rio de Janeiro, encontraram teores de $\mathrm{C}$ significativamente maiores em macroagregados do solo sob vegetação nativa (pasto natural) e SPD, em relação aos encontrados no SPC, para as camadas de $0-5 \mathrm{~cm}(20$, 20 e $15 \mathrm{~g} \mathrm{~kg}^{-1}$, respectivamente) e $10-20 \mathrm{~cm}(17,17 \mathrm{e}$ $12 \mathrm{~g} \mathrm{~kg}^{-1}$, respectivamente). Barreto et al. (2009), em Latossolo Vermelho eutroférrico na Mata Atlântica no Sul do Brasil, encontraram, nos macro, meso e microagregados do solo da camada de $0-5 \mathrm{~cm}$, teores de $\mathrm{C}$ significativamente maiores sob vegetação nativa (52, 87 e $71 \mathrm{~g} \mathrm{~kg}^{-1}$, respectivamente), seguidos dos encontrados em área sob SPD (25, 26 e $30 \mathrm{~g} \mathrm{~kg}^{-1}$, respectivamente), em relação a outra área com SPC (17, 18 e $19 \mathrm{~g} \mathrm{~kg}^{-1}$, respectivamente).

Contudo, ainda não está claro qual classe de agregados do solo é responsável pelo acúmulo de $\mathrm{C} \mathrm{e}$ quais solos, camadas e fatores nos manejos são decisivos nesse processo (Fernández et al., 2010). Os 
resultados desta pesquisa mostraram que, para o Latossolo do presente estudo, a camada de 0-5 cm de solo contribuiu de forma mais expressiva para o acúmulo de $\mathrm{C}$ verificado sob SPD. Isso deve-se, possivelmente, ao maior contato com os resíduos culturais deixados em superfície, que são fonte de matéria orgânica ao solo. A camada de $0-5 \mathrm{~cm}$ foi a mais sensível nos outros usos do solo, e os teores de C foram alterados negativamente no SPC e na PA. Outro relevante resultado é apontado pelos teores de $\mathrm{C}$ contido nos microagregados, os quais, sob SPD, apresentaram teores superiores em relação aos das demais áreas para todas as camadas e, mesmo em menor quantidade que os macro e mesoagregados, foram a classe que mais protegeu o C contra decomposição e, consequentemente, podem ser uma das primeiras classes a acumular esse elemento, com posterior formação de agregados maiores e oclusão de C (Quadro 4).

Assim, o padrão de aumento de C, sobretudo na camada de 0-5 cm de solo, possivelmente esteve relacionado ao enriquecimento desse elemento nos microagregados sob SPD, comparado ao SPC. Nessa classe, acredita-se que a MOS foi inicialmente protegida contra a decomposição e adsorvida aos minerais de argila, com subsequente papel na formação de meso e macroagregados (Anders et al., 2010).
Por sua vez, esses meso e macroagregados proporcionaram acúmulo de $\mathrm{C}$ à medida que a estabilização da MOS foi favorecida pela prática do SPD (predominantemente na camada de 0-5 cm de solo). Por outro lado, essas classes foram alteradas negativamente pelo revolvimento do solo sob sistema convencional de cultivo, com diminuição dos teores de $\mathrm{C}$ em relação aos da $\mathrm{CE}$, enfatizando a maior sensibilidade ao manejo do solo por essas classes de agregados. Os teores de C nos microagregados sob SPC que não apresentaram variação, se comparados aos da $\mathrm{CE}$, reforçam a ideia de que essa classe promove maior proteção ao $\mathrm{C}$ do solo em relação às demais (Quadro 4). Segundo An et al. (2010), o cultivo destrói a estrutura original do solo, aumentando momentaneamente os espaços porosos e, consequentemente, a disponibilidade de oxigênio, levando ao decréscimo da MOS pelo aumento da atividade microbiana.

A falta de diferenças significativas na agregação do solo sob PA em relação a $\mathrm{CE}$, com menores teores de $\mathrm{C}$, indicou que no solo sob PA os macro e mesoagregados não seguiram o mesmo padrão observado no solo sob os diferentes sistemas agrícolas avaliados e, por conseguinte, não seguiram a clássica teoria hierárquica de agregação (Tisdall \& Oades, 1982; Zotarelli et al., 2005).

Quadro 4. Teores de carbono ( $\mathrm{g} \mathrm{kg-1)}$ em agregados de um Latossolo Vermelho distrófico sob diferentes usos e manejos do solo, em Rio Verde (GO)

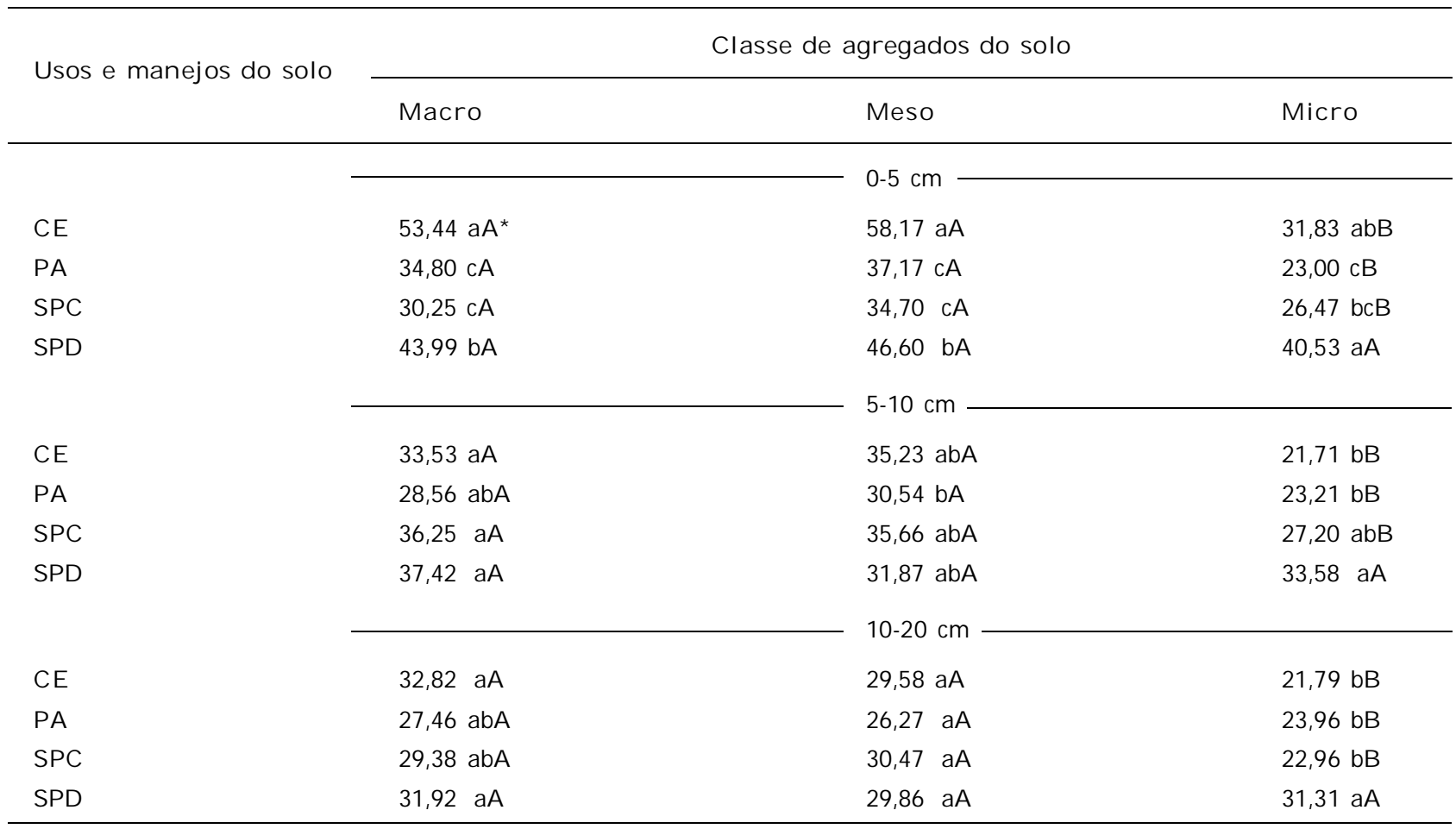

CE: Cerrado nativo; SPC: sistema plantio convencional com 29 anos; SPD: sistema plantio direto com 15 anos; PA: pastagem com 20 anos (previamente cultivada sob SPC por 9 anos). Macro, meso e microagregados: agregados com diâmetro $>2,00$ mm, entre 2,00 e $0,25 \mathrm{~mm}$ e 0,25 e $0,05 \mathrm{~mm}$, respectivamente. Letras iguais nas colunas e letras maiúsculas nas linhas, dentro de cada camada de solo, não diferem a $5 \%$ pelo teste de Tukey. *Valores representam a média $(n=6)$. 
Assim, a área PA, ausente de manejo desde sua introdução, limitou a quantidade de matéria orgânica ao solo, como mostrado em Costa Junior et al. (2011), necessária aos processos de acúmulo de $\mathrm{C}$; e o efeito dos processos físicos (por exemplo, raízes) e mineralógicos de agregação possivelmente foi superior quando comparado ao efeito do acúmulo de C.

A observação supracitada baseia-se na hipótese de que a ausência de manejo tenha levado à queda de produção do pasto (função de um estresse nutricional), que nessa situação pode levar as plantas a tentar compensar a limitada disponibilidade de nutrientes pela exploração de maior volume de solo, favorecendo o acentuado desenvolvimento radicular (Jesko, 1991). Contudo, caso esse estresse nutricional não seja suprimido ou se condições de superpastejo forem impostas, chega-se a uma situação em que a parte aérea da planta não é mais suficiente para manter a demanda fotossintética. Com isso, atinge-se o estádio de degradação propriamente dito, no qual se observa drástica queda da produção, invasão de plantas não palatáveis e desenvolvimento de componentes da fauna do solo que podem se alimentar da matéria orgânica depositada durante esse processo, com consequente diminuição de seus teores (Braz et al., 2004).

A influência de óxidos na agregação do solo é conhecida, tanto que em solos sob clima tropical a MOS e os processos biológicos podem ter papel secundário na formação de agregados (Denef \& Six, 2005; Salton et al., 2008; Fabrizzi et al., 2009). Nessa situação, atua como principal fator de agregação a ligação mineral-mineral por meio de interações eletrostáticas entre óxidos (principalmente de $\mathrm{Fe} \mathrm{e} \mathrm{Al}$ ). Esse fato justificaria a maior agregação do solo sob PA em relação ao SPC e SPD (Figura 2).

Diversos autores têm reportado que a conversão do Cerrado em áreas cultivadas e pastagens conduz à diminuição na quantidade de MOS (Silva et al., 2004; Maia et al., 2009; Siqueira Neto et al., 2009). Por outro lado, uma variedade de opções de manejos do solo (como irrigação, fertilização e introdução de leguminosas) tem mostrado favorecer a manutenção ou até mesmo o acúmulo de $\mathrm{C}$ nesses solos (Silva et al., 2004; Salton et al., 2008). Assim, devido à complexidade da dinâmica do $\mathrm{C}$ no solo, torna-se difícil estabelecer padrões quanto à mudança da vegetação nativa convertida em pastagem.

Maia et al. (2009) afirmam que fatores como variabilidade espacial, tipos de clima e solo, além do histórico de manejo, são apontados como os principais responsáveis por essas diferenças, além do fato de que poucos estudos ainda foram desenvolvidos em solos sob pastagens levando em consideração a agregação do solo e a dinâmica do $\mathrm{C}$.

Nesse sentido, tornam-se necessários novos estudos sobre os teores de $\mathrm{C}$ contido nos agregados do solo, assim como nos diferentes compartimentos da MOS, com o intuito de verificar melhor a dinâmica de agregação principalmente em solos sob pastagem, assim como a influência das diversas vegetações de pastagens existentes.

Os resultados desta pesquisa também mostram as vantagens de utilização de práticas conservacionistas, como o SPD, no aumento da agregação do solo concomitantemente com o aumento dos teores de $\mathrm{C}$ na camada de $0-5 \mathrm{~cm}$ e alertam para a vulnerabilidade do $\mathrm{C}$ do solo sob diferentes sistemas de manejo.

\section{CONCLUSÕES}

1. Solos manejados com sucessão de cultivos (SPD e SPC) apresentam menor quantidade de macroagregados (diâmetro $>2,00 \mathrm{~mm}$ ) e maior de mesoagregados (diâmetro entre 2,00 e $0,25 \mathrm{~mm}$ ) e de microagregados (diâmetro entre 0,25 e 0,05 mm), em comparação ao solo sob vegetação nativa e pastagem, na camada de $0-20 \mathrm{~cm}$.

2. Apenas na camada de $0-5 \mathrm{~cm}$, o solo sob SPD apresenta maior quantidade de macroagregados, se comparado ao solo sob SPC.

3. Os agregados (macro, meso e microagregados) do solo, na camada de $0-20 \mathrm{~cm}$, sob vegetação nativa e SPD apresentam teores de $\mathrm{C}$ similares e superiores aos do solo sob SPC e PA.

4. Os microagregados oferecem maior proteção ao $\mathrm{C}$ do solo, enquanto os macro e mesoagregados são mais sensíveis, sendo alterados negativamente pelo revolvimento do solo sob SPC e pela ausência de manejo da PA.

5. A ausência de manejo da pastagem proporciona agregados do solo em proporções semelhantes e com menores teores de $\mathrm{C}$, comparada ao solo sob vegetação nativa, sugerindo dinâmica de agregação diferenciada e que merece atenção de novas pesquisas.

\section{AGRADECIMENTOS}

À Fundação Agrisus (164/04) e à FAPESP (2004/ 15538-7; 06/52201-6), pelo apoio financeiro. Aos proprietários das fazendas Alvorada, Barra Grande do Rio Verdinho e Dois-J-1, em Rio Verde (GO), pela contribuição no desenvolvimento deste trabalho de pesquisa.

\section{LITERATURA CITADA}

AN, S.; MENTLER, A.; MAYER, H. \& BLUM, W.E.H. Soil aggregation, aggregate stability, organic carbon and nitrogen in different soil aggregate fractions under forest and shrub vegetation on the Loess Plateau, China. Catena, 81:226-233, 2010. 
ANDERS, M.M.; BECK, P.A.; WATKINS, B.K.; GUNTER, S.A.; LUSBY, K.S. \& HUBBELL, D.S. Soil aggregates and their associated carbon and nitrogen content in winter annual pastures. Soil Water Manag. Conserv., 74:1339-1347, 2010.

BARRETO, R.C.; MADARI, B.E.; MADDOCK, J.E.L.; MACHADO, P.L.O.A.; TORRES, E.; FRANCHINI, J. \& COSTA, A.R. The impact of soil management on aggregation, carbon stabilization and carbon loss as $\mathrm{CO}_{2}$ in the surface layer of a Rhodic Ferralsol in Southern Brazil Agric. Ecosyst. Environ., 132:243-251, 2009.

BAYER, C.; MARTIN-NETO, L.; MIELNICZUC, J.; PAVINATO, A. \& DIECKOW, J. Carbon sequestration in two Brazilian Cerrado soils under no-till. Soil Tillage Res., 86:237-245, 2006.

BLAKE, G.R. \& HARTGE, K.H. Bulk density. In: KLUTE, A. (Ed). Method soil analysis. 2.ed. Madison, ASA, 1986. Pt.1. 364-367p. (Agronomy, 9)

BRASIL, Ministério das Minas e Energia. Projeto RADAMBRASH: folha SE 22-x-r Rio Verde-GO: geologia, geomorfologia, pedologia, vegetação,uso potencial da terra. Rio de Janeiro, 1981. (Levantamento dos recursos naturais, 25)

BRAZ, S.P.; URQUIAGA, S.; ALVES, B.J.R. \& BODDEY, R.M. Degradação de pastagens, matéria orgânica do solo e a recuperação do potencial produtivo em sistemas de baixo input tecnológico na região dos cerrados. Seropédica, Embrapa, 2004. (Circular Técnica, 9)

BRONICK, C.J. \& LAL, R. Soil structure and management: A review. Geoderma, 124:3-22, 2005.

CORRÊA, J.C. Efeito de sistemas de cultivo na estabilidade de agregados de um Latossolo Vermelho-Amarelo em Querência, MT. Pesq. Agropec. Bras., 37:203-209, 2002.

COSTA JUNIOR, C.; PÍCCOLO, M.C.; SIQUEIRA NETO, M.; CAMARGO, P.B.; CERRI, C.C. \& BERNOUX, M. Carbono total e $\mathrm{a}^{13} \mathrm{C}$ em agregados do solo sob vegetação nativa e pastagem no bioma Cerrado. R. Bras. Ci. Solo, 35:1241$1252,2011$.

DE GRYZE, S.; BOSSUYT, H.; SIX, J.; van MEIRVENNE, M.; GOVERS, G. \& MERCKX, R. Factors controlling aggregation in a minimum and a conventionally tilled undulating field. Eur. J. Soil Sci., 58:1017-1026, 2008.

DENEF, K. \& SIX, J. Clay mineralogy determines the importance of biological versus abiotic processes for macroaggregate formation and stabilization. Eur. J. Soil Sci., 56:469-479, 2005.

EITEN, G. The cerrado vegetation of Brazil. Bot. Rev., 38:201$341,1972$.

EMPRESA BRASILEIRA DE PESQUISA AGROPECUÁRIA EMBRAPA. Manual de métodos de análise do solo, 2a ed, Rio de Janeiro, 1997. 212p.

EMPRESA BRASILEIRA DE PESQUISA AGROPECUÁRIA EMBRAPA. Centro Nacional de Pesquisa de Solos. Sistema brasileiro de classificação de solos. 2.ed. Rio de Janeiro, 2006. 306p.

FABRIZZI, K.P.; MORON, A. \& GARCÍA, F.O. Soil Carbon and nitrogen organic fractions in degraded vs. non-degraded Mollisols in Argentina. Soil Sci. Soc. Am. J., 67:1831-1841, 2003.
FABRIZZI, K.P.; RICE, C.W.; AMADO, T.J.C.; FIORIN, J.; BARBAGELATA, P. \& MELCHIORI, R. Protection of soil organic $\mathrm{C}$ and $\mathrm{N}$ in temperate and tropical soils: Effect of native and agroecosystems. Biogeochemist., 92:129-143, 2009.

FERNÁNDEZ, R.; QUIROGA, A.; ZORATI, C. \& NOELLEMEYER, E. Carbon contents and respiration rates of aggregate size fractions under no-till and conventional tillage. Soil Tillage Res., 109:103-109, 2010.

GREEN, V.S.; CAVIGELLI, M.A.; DAO, T.H. \& FLANAGAN, D.C. Soil physical properties and aggregate- associated $\mathrm{C}, \mathrm{N}$ and $\mathrm{P}$ distributions in organic and conventional cropping systems. Soil Sci. J., 170:822-831, 2005.

HAYNES, R.J. \& BEARE, M.H. Influence of six crop species on aggregate stability and some labile organic matter fractions. Soil Biol. Biochem., 29:1647-1653, 1997.

HUANG, L.; WANG, C.Y.; TAN, W.F.; HU, H.Q.; CAI, C.F. \& WANG, M.K. Distribution of organic matter in aggregates of eroded Ultisols, Central China. Soil Tillage Res., 108:5967, 2010.

JEŠKO, T. The root as an internal part of the plant. In: KOLEK, J. \& KOZINKA, V., eds. Physiology of the plant root system. New York, Kluwer, 1991. p.1-30.

KEMPER, W.D. \& CHEPIL, W.S. Size distribution of aggregates. In: BLACK, C.A., ed. Methods of soil analysis. Physical and mineralogical properties, including statistics of measurements and sampling, Madison, America Society Agronomy, 1965. Part 1. p.499-510. (Agronomy, 9)

LI, X.G.; WANG, Z.F.; MA, Q.F. \& LI, F.M. Crop cultivation and intensive grazing affect organic $\mathrm{C}$ pools and aggregate stability in arid grassland soil. Soil Tillage Res., 95:172$181,2007$.

MADARI, B.E.; MACHADO, P.L.O.A.; TORRES, E.; ANDRADE, A.G. \& VALENCIA, L.I.O. No tillage and crop rotation effects on soil aggregation and organic carbon in a Rhodic Ferralsol from southern Brazil. Soil Tillage Res., 80:185$200,2005$.

MAIA, S.M.F.; OGLE, S.M.; CERRI, C.E.P. \& CERRI, C.C. Effect of grassland management on soil carbon sequestration in Rondônia and Mato Grosso states, Brazil. Geoderma, 149:84-91, 2009.

MARTINS, M.R.; CORA, J.E.; JORGE, R.F. \& MARCELO, A.V. Crop type influences soil aggregation and organic matter under no-tillage. Soil Tillage Res., 104:22-29, 2009.

MUGGLER C.C.; van GRIETHUYSEN, C.P.; BUURMAN, P. \& PAPE, T. Aggregation, organic matter, and iron oxide morphology in Oxisols from Minas Gerais, Brazil. Soil Sci. J., 164:759-770, 1999.

MULUMBA, L.N. \& LAL, R. Mulching effects on select soil properties. Soil Tillage Res., 98:106-111, 2008.

MUNSELL COMPANY INC. Munsell Soil Color Charts. Munsell Company Inc, Baltimore,1954.

NOELLEMEYER, E.; FRANK, F.; ALVAREZ, C.; MORAZZO, G. \& QUIROGA, A. Carbon contents and aggregation related to soil physical and biological properties under a land-use sequence in the semiarid region of central Argentina. Soil Tillage Res., 99:179-190, 2008. 
OGLE, S.M.; BREIDT, F.J.; EVE, M. \& PAUSTIAN, K. Uncertainty in estimating land use and management impacts on soil organic carbon storage for US agricultural Lands between 1982-1997. Global Change Biol., 9:1521$1542,2003$.

PINHEIRO, E.F.M.; PEREIRA, M.G. \& ANJOS, L.H.C. Aggregate distribuition and soil organic matter under different tillage systems for vegetable crops in a Red Latosol from Brazil. Soil Tillage Res., 77:79-84, 2004.

SALTON, J.C.; MIELNICZUK, J.; BAYER, C.; BOENI, M.; CONCEIÇÃO, P.C.; FABRÍCIO, A.C.; MACEDO, M.C.M. \& BROCH, D.L. Agregação e estabilidade de agregados do solo em sistemas agropecuários em Mato Grosso do Sul. R. Bras. Ci. Solo, 32:11-21, 2008.

SAS Institute. SAS 9.1.3 Service Pack 3. Cary, 2003. Disponível em: <http://www.sas.com>.

SILVA, I.F. \& MIELNICZUK, J. Avaliação do estado de agregação do solo afetado pelo uso agrícola. R. Bras. Ci. Solo, 21:313-319, 1997.

SILVA, J.E.; RESCK, D.V.S.; CORAZZA, E.J. \& VIVALDI, L. Carbon storage under cultivated pastures in a clayey Oxisol in the Cerrado Region, Brazil. Agric. Ecos. Environ., 103:357-363, 2004.

SIQUEIRA NETO, M.; PÍCCOLO, M.C.; SCOPEL, E.; COSTA JUNIOR, C.; CERRI, C.C. \& BERNOUX, M. Carbono total e atributos químicos com diferentes usos do solo no Cerrado. Acta Sci. Agron., 31:709-717, 2009.

SIQUEIRA NETO, M.; SCOPEL, E.; CORBEELS, M.; CARDOSO, A.N.; DOUZET, J.; FELLER, C.; PÍCCOLO, M.C.; CERRI, C.C. \& BERNOUX, M. Soil carbon stocks under no-tillage mulch-based cropping systems in the Brazilian Cerrado: An on-farm synchronic assessment. Soil Tillage Res., 110:187-195, 2010.
SIX, J. \& JASTROW, J.D. Soil organic matter turnover. In: LAL, R., ed. Encyclopaedia of soil science. New York, Marcel Dekker, 2002. p.936-942.

SIX, J.; BOSSUYT, H.; GRYZE, S. \& DENEF, K. A history of research on the link between (micro) aggregates, soil biota, and soil organic matter dynamics. Soil Tillage Res., 79:7-31, 2004.

SIX, J.; PAUSTIAN, K.; ELLIOTT, E.T. \& COMBRINK, C. Soil structure and soil organic matter: I. Distribution of aggregate size classes and aggregate associated carbon. Soil Sci. Soc. Am. J., 64:681-689, 2000.

SIX, J.; ELLIOT, E.T.; PAUSTIAN, K. \& DORAN, J.W. Aggregation and soil organic matter accumulation in cultivated and native grassland soils. Soil Sci. Soc. Am. J., 62:1367-1377, 1998.

SIX, J.; FREY, S.D.; THIET, R.K. \& BATTEN, K.M. Bacterial and fungal contributions to carbon sequestration in agroecosystems. Soil Sci. Soc. Am. J., 70:555-569, 2006.

STEVENSON, F.J. Humus chemistry: Genesis, composition, reactions. 2.ed. New York, John Wiley \& Sons, 1994.

TISDALL, J.M. \& OADES, L.M. Organic matter and waterstable aggregates in soil. Soil Sci. J., 33:141-163, 1982.

ZOTARELLI, L.; ALVES, B.; URQUIAGA, S.; BODDEY, R. \& SIX, J. Impact of tillage and crop rotation on aggregateassociated carbon in two Oxisols. Soil Sci. Soc. Am. J., 69:482-491, 2007.

ZORATELLI, L.; ALVES, B.J.R.; URQUIAGA, S.; TORRES, E.; DOS SANTOS, H.P.; PAUSTIAN, K.; BODDEY, R.M. \& SIX, J. Impact of tillage and crop rotation on aggregateassociated carbon in two Oxisols. Soil Sci. Soc. Am. J., 69:482-491, 2005. 
R. Bras. Ci. Solo, 36:1311-1321 